\title{
Un espoir thérapeutique dans la maladie d'Alzheimer, ou peut-on traiter le mal par le mal ?
}

II apparaît de plus en plus vraisemblable que le dépôt cérébral de peptide $\beta$ amyloïde insoluble $A \beta$ constitue une étape-clé du processus pathologique de la maladie d'Alzheimer. Ce peptide très hydrophobe a la capacité de s'agréger spontanément in vitro et d'être neurotoxique in vitro et in vivo. Son agrégation sous forme de plaques séniles constitue l'une des deux signatures neuropathologiques de cette maladie, avec les protéines tau hyperphosphorylées, constituant des dégénérescences neurofibrillaires [1]. Le peptide amyloïde existe sous plusieurs formes, de 39 à 43 acides aminés. Ces formes dérivent du clivage du précurseur de la protéine amyloïde (APP). Les mutations affectant les sites de clivage de I'APP, ou celles des gènes des présénilines 1 et 2 , provoquent une accumulation du peptide $A \beta$, essentiellement celui de 42 acides aminés ( $A \beta 42)$, et sont responsables de rares formes héréditaires précoces de la maladie $(\mathrm{m} / \mathrm{s}$ $1998, n^{\circ} 4$, p. 486). Les taux plasmatiques d'A $\beta 42$ sont également élevés chez les apparentés des patients atteints des formes tardives de la maladie.

C'est à partir de ce dernier critère que vient d'être identifié un nouveau locus de susceptibilité génétique sur le chromosome 10 [2, 3]. L'implication de cette région du chromosome 10 est confirmée dans le même numéro de Science par une autre étude qui révèle un impact d'amplitude similaire à la présence de I'allèle $\varepsilon 4$ de l'apolipoprotéine $E$, le facteur génétique de susceptibilité le mieux caractérisé jusqu'à présent [4]. Le gène impliqué sur le chromosome 10 serait donc un facteur de risque majeur des formes tardives de la MA et modifierait le métabolisme de I'A $\beta 42$. A environ $30 \mathrm{~cm}$ de la région en question, une autre liaison génétique recouvrant le locus de I'IDE (insulin degrading enzyme) a été également publiée dans la même livraison de Science [5]. Sachant que la localisation estimée par les études de liaisons génétiques peut facilement varier de 20 à $30 \mathrm{cM}$, il se pourrait que le gène de l'IDE soit lui même le gène délétère; $d$ 'autant plus que cette enzyme sécrétée par les neurones et la microglie peut dégrader le peptide $A \beta$ [6]. La diminution d'activité enzymatique IDE observée dans le tissu cérébral des patients pourrait entraîner une augmentation des taux d'A $\beta$. La recherche de mutations dans le gène de l'IDE est en cours.

A côté des études génétiques, l'obtention de modèles animaux de maladie d'Alzheimer représente une étape incontournable pour la compréhension de la physiopathologie de la maladie et la recherche de thérapeutiques. Ainsi, des souris transgéniques surexprimant une forme mutée de l'APP humaine (PDAPP) présentent une accumulation de peptide $A \beta$ qui a pour conséquence I'apparition croissante avec l'âge de dépôts amyloïdes, de neurites dystrophiques et d'une gliose réactionnelle [7]. De plus, ces souris développent assez rapidement des déficits mnésiques marqués évalués par des tests en labyrinthe aquatique. Chen et al., en raffinant le test du labyrinthe aquatique, viennent de préciser la chronologie d'apparition de ces déficits. En effet, certains déficits d'apprentissage de localisation spatiale corrèlent avec la quantité de dépôts amyloïdes dans I'hippocampe et s'accentuent avec l'âge des souris PDAPP [8], tandis que les déficits d'apprentissage constitutionnels sont indépendants de l'âge et de la quantité de peptides $A \beta$ déposés. L'ensemble de ces altérations mime donc assez bien les symptômes et une partie des caractéristiques de la maladie d'Alzheimer.

Ce modèle animal paraît donc très adapté pour tester l'impact de molécules visant à prévenir le déclin cognitif et l'évolution du processus pathologique. De manière tout à fait étonnante, l'injection du peptide fibrillaire $A \beta 42$ entraîne la production d'anticorps anti-A $\beta$ et prévient la formation des dépôts amyloïdes, des neurites dystrophiques et de l'astrogliose chez les souris PDAPP âgées de 6 semaines encore indemnes de toute lésion [7]. Si l'immunisation est effectuée à l'âge de 11 mois, alors que les dépôts amyloïdes sont déjà bien installés, on observe un ralentissement de leur progression et une réduction de plus de $90 \%$ de leur étendue. Bien que moins spectaculaire, une immunisation par le peptide $A \beta 40$ réalisée par voie nasale confirme la réduction du processus pathologique. Ces expériences semblent très prometteuses pour la mise au point d'un traitement efficace de la maladie. Toutefois, la faible immunogénicité des fibrilles d'A $\beta$ nécessite des administrations répétées afin d'obtenir un titre élevé d'anticorps. Par ailleurs, il est important de s'assurer que l'immunisation par un composé toxique sous forme de fibrilles $A \beta$ n'entraîne pas de complications immunitaires ou n'interfère pas avec le fonctionnement normal du cerveau.

Deux études réalisées sur deux autres modèles murins de maladie d'Alzheimer semblent confirmer les effets bénéfiques d'une immunisation par 
I'A $\beta$ sur les capacités d'apprentissage et de mémoire $[9,10]$. Dans la première, les souris étant transgéniques pour une autre forme mutée d'APP humaine, le traitement des animaux par 5 injections d'A $\beta 42$ humaine jusqu'à l'âge de 20 semaines permet une nette amélioration de leurs capacités d'apprentissage (essentiellement observée à partir de la $23^{\mathrm{e}}$ semaine) comparée à des souris ayant reçu un autre immunogène. Cependant, le rétablissement n'est pas total puisque les souris transgéniques immunisées par l'A $\beta$ ont des performances moindres que les souris sauvages. Des résultats similaires sont obtenus dans un autre modèle (surexpression des formes mutées humaines de I'APP et de préséniline1), caractérisé par une progression plus lente de la maladie. Après 5 injections effectuées avant le $12^{\mathrm{e}}$ mois, les capacités de mémoire et d'apprentissage de ces souris sont semblables à celles des souris saines, alors qu'après 9 immunisations, vers 15 mois, les souris ont un score se rapprochant de ceux des témoins.

$L$ a vaccination par I'A 342 humaine chez la souris permettrait donc de restituer une partie des fonctions cognitives perdues, sans causer de réaction inflammatoire génératrice de dommages sur le fonctionnement cérébral. Si ces résultats apparaissent spectaculaires, leur interprétation reste très parcellaire. Quels sont les mécanismes mis en jeu ? Les améliorations semblent liées à une réduction de $50 \%$ du nombre et de la taille des plaques séniles sans modification des taux cérébraux d'holoprotéine APP, de fragments APP solubles et carboxy-terminaux. Ces données suggèrent que le nombre de plaques pourrait être un élément important du déclin cognitif. Cependant, chez les souris transgéniques, certains dysfonctionnements cognitifs apparaissent avant les dépôts d'amyloïdes. De plus, chez l'homme, les plaques séniles et la démence sont très mal corrélées. On peut formuler I'hypothèse selon laquelle la quantité $d^{\prime} A \beta$ libre pourrait jouer un rôle néfaste sur les fonctions cognitives. Le rôle de la plaque pourrait alors être de séquestrer certaines formes d'A $\beta$ toxiques en une structure inerte ce qui conduirait à préserver les fonctions cérébrales. Suite à la vaccination, une infime partie $(0,1 \%)$ des anticorps, ayant traversé la barrière hémato-encéphalique [11], pourrait alors interagir avec l'A $\beta$ des plaques, désagréger l'amyloïde préformée et inhiber la formation des fibrilles [12]. Le peptide $A \beta$ pourrait ensuite être dégradé par la microglie via la voie de signalisation du récepteur Fc des vésicules de phagocytose [11]. Une autre hypothèse serait l'infiltration des monocytes conduisant à une activation de la microglie.

Peut-on espérer un mécanisme similaire chez l'homme? L'immunisation chez I'homme sera-t-elle alors aussi efficace que chez l'animal ? De même, l'élimination des plaques sera-t-elle aussi facile chez l'homme, les constituants des plaques et la proportion de polymères d'A $\beta$ différant entre I'homme et le modèle animal? Faudra-t-il également administrer des adjuvants comme chez la souris ? Les doses d'antigènes à utiliser serontelles suffisantes pour obtenir un titre en anticorps élevé ? La réponse inflammatoire sera-telle aussi efficace d'un individu à l'autre, puisque la prise en charge de la toxicité de l'A $\beta$ est fonction du patrimoine génétique de l'individu ? Enfin, le modèle murin ne présente pas de dégénérescence neurofibrillaire, la deuxième caractéristique neuropathologique de la maladie d'Alzheimer qui, contrairement aux plaques séniles, apparaissent bien corrélées avec l'état démentiel des patients... On voit qu'un certain nombre de questions subsiste mais le peptide $A \beta$, si souvent incriminé dans le développement de la maladie d'Alzheimer, est aujourd'hui une source d'espoir pour la thérapeutique, d'autant plus que les premiers essais réalisés aux États-U nis montrent I'innocuité du vaccin chez l'homme.

1. Tranchant C. Protéines TAU et maladies neurologiques. M ed Sci 1997 ; 13: 989-97.

2. Ertekin-Taner N, Graff-Radford N, Younkin LH, et al. Linkage of plasma Abeta42 to a quantitative locus on chromosome 10 in late-onset Alzheimer's disease pedigrees. Science 2000; 290 : 2303-4.

3. Kehoe $P$, Wavrant-De Vrieze $F$, et al. A full genome scan for late onset Alzheimer's disease. Hum M ol Genet 1999; 8 : 237-45.

4. Myers A, Holmans P, Marshall H, et al. Susceptibility locus for Alzheimer's disease on chromosome 10. Science 2000; 290: 2304-5.

5. Bertram L, Blacker D, Mullin K, et al. Evidence for genetic linkage of Alzheimer's disease to chromosome 10q. Science 2000; 290: 2302-3.

6. Qiu WQ, Ye Z, Kholodenko D, Seubert P, Selkoe DJ. Degradation of amyloid-protein by a metalloprotease secreted by microglia and other neural and non-neural cells. J Biol Chem 1997; 272: 6641-6.

7. Schenk D, Barbour R, Dunn W, et al. Immunization with amyloid-beta attenuates Alzheimerdisease-like pathology in the PDAPP mouse. Nature 1999; 400: 173-7.

8. Chen G, Chen KS, Knox J, et al. A learning deficit related to age and beta-amyloid plaques in a mouse model of Alzheimer's disease. Nature 2000; 408: 975-9.

9. Morgan D, Diamond DM, Gottschall PE, et al. A beta peptide vaccination prevents memory loss in an animal model of Alzheimer's disease. Nature $2000 ; 408$ : 982-5.

10. Janus C, Pearson J, McLaurin J, et al. A beta peptide immunization reduces behavioural impairment and plaques in a model of Alzheimer's disease. Nature 2000; 408: 979-82.

11. Bard F, Cannon C, Barbour R, et al. Peripherally administered antibodies against amyloid beta-peptide enter the central nervous system and reduce pathology in a mouse model of Alzheimer disease. Nat M ed 2000; 6: 916-9.

12. Solomon B, Koppel R, Frankel D, Hanan-Aharon $E$. Disaggregation of Alzheimer beta-amyloid by site-directed mAb. Proc Natl Acad Sci USA 1997; 94 : 4109-12.

\section{Marie-C hristine Chartier-H arlin Louisa Araria-G oumidi}

Inserm U. 508, Institut Pasteur de Lille, 1, rue du Pr.-Calmette, BP 245, 59019 Lille Cedex, France. 